\title{
Acknowledgments
}

HE BOOK GREW OUT OF A CONFERENCE ON "THE
PLA's Role in National Security Policy-Making" co-sponsored by Taiwan's Council of Advanced Policy Studies (CAPS), RAND, the Carnegie Endowment for International Peace (CEIP), and National Defense University (NDU). The editors gratefully acknowledge the intellectual contributions of co-organizers Arthur Shu-fan Ding (then at CAPS, now at Chengchi University's Institute of International Relations) and Michael Swaine (CEIP) in shaping the agenda and recruiting paper writers, discussants, and panelists, and securing the financial support of the four sponsoring organizations.

Conference participants Ken Allen, Jean-Pierre Cabestan, Chia-sheng Chen, Roger Cliff, Bernard Cole, Cortez Cooper, Scott Harold, Alexander Huang, Brian Lafferty, Chong-pin Lin, Kim Taeho, Ma Chengkun, Chengting Tsai, Shinji Yamaguchi, and Suisheng Zhao all provided comments or participated in discussions that improved the chapters in this book. Andrew Nien-Dzu Yang, Yi-su Yang, and Polly Shen (CAPS), Cortez Cooper, Scott Harold, and Lyle Morris (RAND), and Michael Glosny, Isaac Kardon, Deborah Jefferson, and Don Mosser (NDU) all provided support in organizing the original conference.

The editors thank the two anonymous reviewers who provided much useful advice in shaping the book and updating the individual chapters. Molly Murphy and Deborah Jefferson provided assistance in the legal and administrative aspects of publishing this book. Joseph Kettel helped 
produce summaries of the chapters for the introduction. Drew Casey provided invaluable assistance in reformatting the chapters, tracking down stray references, and standardizing the English and Chinese language endnotes.

Geoffrey Burn and James Holt at Stanford University Press deserve special thanks for guiding the book through the approval and publication process. 


\section{PLA INFLUENCE ON CHINA'S \\ NATIONAL SECURITY \\ POLICYMAKING}


